\title{
Capmatinib and gefitinib combination therapy: will EGFR-mutated MET-dysregulated NSCLC "capitulate"?
}

\author{
Brian Ko ${ }^{1}$, Balazs Halmos ${ }^{2}$ \\ ${ }^{1}$ Department of Medicine, ${ }^{2}$ Department of Oncology, Montefiore Medical Center, Albert Einstein College of Medicine, Bronx, NY 10461, USA \\ Correspondence to: Balazs Halmos, MD. Department of Oncology, Montefiore Medical Center, Albert Einstein College of Medicine, Bronx, NY \\ 10461, USA. Email: bahalmos@montefiore.org. \\ Comment on: Wu YL, Zhang L, Kim DW, et al. Phase Ib/II Study of Capmatinib (INC280) Plus Gefitinib After Failure of Epidermal Growth Factor \\ Receptor (EGFR) Inhibitor Therapy in Patients With EGFR-Mutated, MET Factor-Dysregulated Non-Small-Cell Lung Cancer. J Clin Oncol \\ 2018. [Epub ahead of print].
}

Submitted Nov 30, 2018. Accepted for publication Dec 12, 2018.

doi: $10.21037 /$ tlcr.2018.12.05

View this article at: http://dx.doi.org/10.21037/tlcr.2018.12.05

The mesenchymal-epithelial transition factor (MET)/ hepatocyte growth factor (HGF) axis is a key pathway in acquired resistance against epidermal growth factor receptor (EGFR) tyrosine kinase inhibitor (TKI) therapy in EGFR-mutated non-small cell lung cancer (NSCLC). The binding of the cognate HGF ligand to MET induces receptor activation, promoting cell proliferation, cell invasion, and cell survival in multiple preclinical cancer models (1). Evidence of MET dysregulation conferring resistance to EGFR TKI therapy was identified as early as 2007 when Engelman and colleagues demonstrated that focal MET amplification drives ERBB3-dependent activation of PI3K and subsequent resistance to gefitinib. Crucially, MET inhibitor monotherapy was not sufficient to overcome gefitinib resistance; combined treatment with a MET inhibitor and gefitinib was required, suggesting that selective pressure exerted by gefitinib causes cancer cells to adapt so that sustained downstream signaling of either the EGFR pathway or MET pathway is sufficient for survival (2), necessitating the concurrent use of both an EGFR inhibitor and a MET inhibitor. These early observations would herald the paramount importance of carefully selecting EGFRmutated NSCLC patients with documented acquired resistance to primary EGFR TKI therapy in the clinical development of MET inhibitors, as earlier disappointingly negative clinical studies investigating MET inhibitors largely featured unselected NSCLC patient populations.

Further preclinical studies have demonstrated that MET can engage several other cell surface oncogenic receptor tyrosine kinases including AXL and EPHA1 and can induce
EGFR to stabilize potentially oncogenic multiprotein complexes with binding partners including CDCP1 and JAK in multiple carcinoma cell lines. In this context, EGFR downstream signaling becomes independent of its kinase function and competitive ATP binding, conferring resistance to ATP-mimetic EGFR TKI monotherapy (3). MET activity can be dysregulated in a variety of ways such as MET or HGF overexpression, MET gene amplification or high gene copy number (GCN) (4). The frequency of MET dysregulation has ranged from $5-26 \%$ of NSCLC with acquired resistance to EGFR inhibitors dependent on methods used and thereby constitutes the second most common validated mechanism of EGFR resistance next to EGFR T790M (2,5-8). Specifically, MET amplification appears to be a more frequent resistance mechanism to the third-generation EGFR TKI osimertinib compared to first-generation EGFR TKIs (9). As such, the increasing use of osimertinib for first-line treatment of patients with metastatic NSCLC harboring EGFR exon 19 deletions or exon 21 L858R mutations may dramatically increase the clinical need for efficacious MET inhibitors. Beyond the combination of first-generation EGFR TKIs with MET inhibitors, which the current study addresses with gefitinib and capmatinib, there are early case reports suggesting that the combination of osimertinib and crizotinib has clinical activity against $E G F R$-mutated NSCLC with acquired resistance to EGFR TKI therapy $(10,11)$.

Small molecule inhibitors of MET range considerably in structure, mechanism of action and selectivity. Class I inhibitors tend to be more selective for MET and bind to 
the activation loop adjacent to the hinge region of MET with a U-shaped conformation (12). Class II inhibitors are usually less selective for MET and when binding to MET span the area from the hinge region to the C-helix (12). Both classes I and II are ATP-competitive, but there are other small molecule MET inhibitors, such as tivantinib, that are ATP non-competitive and have high affinity for unphosphorylated MET, strongly inhibiting MET autophosphorylation and subsequent receptor activation (13). Capmatinib is an orally bioavailable and potent type I MET inhibitor with greater than 10,000-fold selectivity for MET over other tested kinases (14). In 2014, acceptable safety and promising preliminary clinical activity of capmatinib in combination with gefitinib was already reported from a single-arm phase Ib/II study which enrolled adult patients with locally advanced or metastatic EGFR-mutated METdysregulated NSCLC with progression after EGFR TKI treatment (15). Recently, eagerly awaited results of the entire study have reported out (16).

Regarding MET biomarker selection for the current study, phase $\mathrm{Ib}$ patients were required to have MET amplification defined as a MET GCN greater than or equal to five and/or a $M E T /$ centromere ratio greater than or equal to 2.0, or MET overexpression defined as greater than or equal to $50 \%$ of tumor cells with moderate or strong staining intensity. After completing the current phase $\mathrm{Ib}$ study, the investigators determined the recommended phase II dose of capmatinib to be $400 \mathrm{mg}$ by mouth twice daily to be administered with gefitinib $250 \mathrm{mg}$ by mouth daily. For phase II, patients were initially required to have a MET GCN greater than or equal to 5 by FISH or $50 \%$ of tumor cells with MET immunohistochemistry (IHC) $2+/ 3+$; this was subsequently amended to a MET GCN greater than or equal to four or $50 \%$ of tumor cells with MET IHC $3+$. IHC was used to assess changes in downstream pathway activation, such as phosphorylated (p)-MET, p-ERK, p-AKT, and p-S6, in both pre and post-treatment samples. Although both realtime polymerase chain reaction (PCR) and fluorescence in situ hybridization (FISH) can accurately assess copy numbers of $M E T$, real-time PCR cannot distinguish between true MET amplification and polysomy (4). MET IHC tends to incorrectly overestimate MET amplification, while FISH and MET/CEP7 ratio can more accurately determine true genomic amplification (5). Thus, MET gene copy number (GCN) was determined with FISH and when additional tumor tissue was available, nextgeneration sequencing was performed in order to identify and document specific MET mutations (though specific mutations were not mentioned in the manuscript). Other potential predictive biomarkers include MET/CEP7 ratio, but the investigators found that responses corresponded better with MET GCN in the current study.

The primary end point for the phase II portion was overall response rate (ORR) as determined by Response Evaluation Criteria in Solid Tumors (RECIST) version 1.1, which was $29 \%$ (29 of 100 patients) in the phase II study and $27 \%$ across both phase Ib and phase II studies. While the overall response rate of $29 \%$ in the phase II portion seems lower than anticipated, an exploratory subgroup analysis involving a biomarker enriched group of 36 patients with a $M E T$ GCN of six or greater, the response rate was $47 \%$ (17 of 36 patients). Progression-free survival (PFS) varied by MET GCN; for MET GCN less than four PFS was 3.9 months, for MET GCN in between four and six PFS was 5.4 months and for MET GCN greater than or equal to six PFS was 5.5 months.

The combination of capmatinib and gefitinib was relatively tolerable with treatment-related grade $3 / 4$ adverse events (AEs) in 46 of 161 (29\%) patients and treatment discontinuation in 27 of $161(17 \%)$ patients. No notable drug-drug interactions were observed between capmatinib and gefitinib. While the frequency of grade $3 / 4 \mathrm{AEs}$ is quite high, this compares favorably to treatment-related adverse events with other selective MET inhibitors in clinical development including tepotinib, volitinib, and glesatinib. For comparison, in the recent GEOMETRY mono-1 trial $84 \%$ of patients that received capmatinib had an $\mathrm{AE}$ with one-third of patients having grade 3/4 AEs.

The current study suggests that there is a therapeutic ceiling for the combination of capmatinib and gefitinib in EGFR-mutated MET-dysregulated NSCLC. IHC was used to assess changes in activation of key downstream markers, including p-MET, p-ERK, p-AKT, and p-S6, and at the doses used, excellent inhibition of the pathway could be demonstrated. Despite that, even in the subgroup of patients with a MET GCN greater than or equal to six with an ORR of $47 \%$, PFS was quite short-lived at only 5.5 months without long-term responders. The lack of durable responses raises concerns about possible tumor heterogeneity leading to differential responses to therapy as well as rapid acquired resistance through unknown mechanisms despite concurrent and potent EGFR and MET blockade.

Another drawback is that while the current study addresses the combination of gefitinib and capmatinib, in current clinical practice osimertinib has supplanted gefitinib for the first-line treatment of patients with metastatic 
Table 1 Selected clinical studies of MET inhibitors in NSCLC

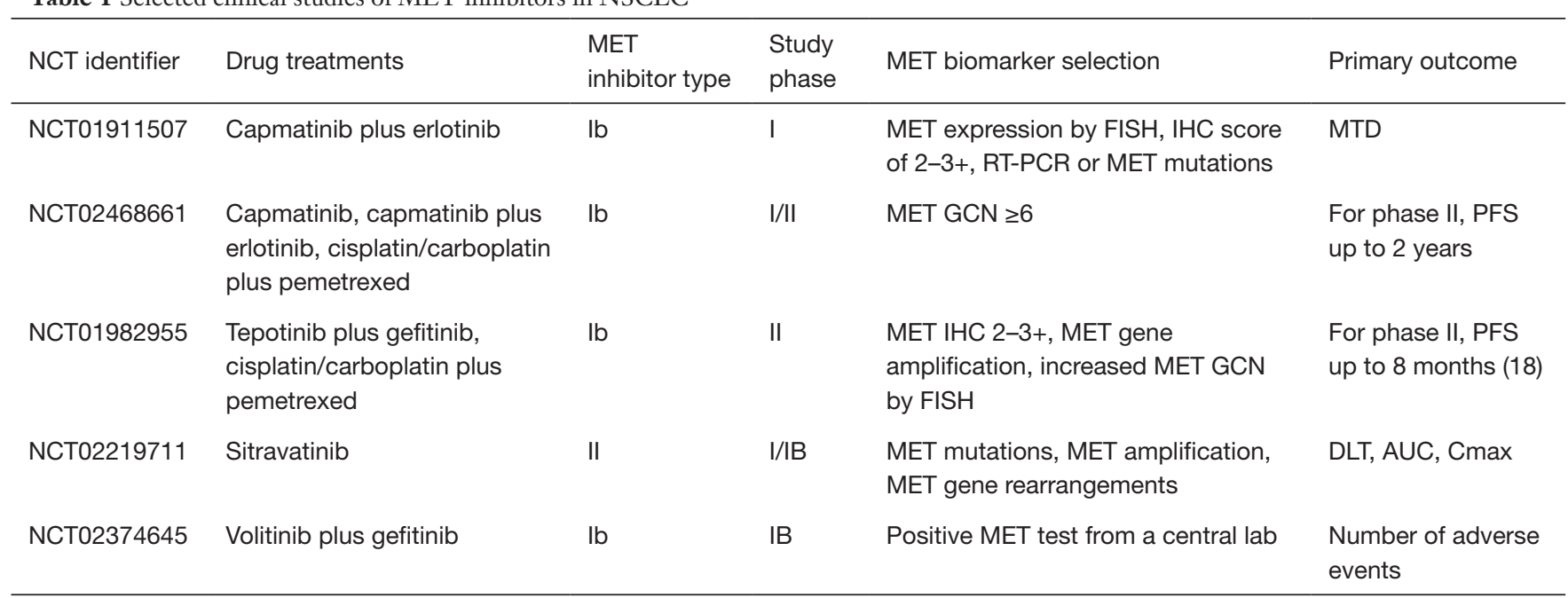

MET, mesenchymal-epithelial transition factor; NSCLC, non-small cell lung cancer; NCT, National Clinical Trial; FISH, fluorescence in situ hybridization; IHC, immunohistochemistry; RT-PCR, reverse-transcriptase polymerase chain reaction; MTD, maximum tolerated dose; GCN, gene copy number; PFS, progression-free survival; DLT, dose-limiting toxicity; AUC, area under the curve; Cmax, peak serum concentration.

NSCLC harboring EGFR exon 19 deletions or exon 21 L858R mutations; given the rapidly changing treatment landscape for EGFR-mutated NSCLC, choosing the most promising interventional drug combination and the most appropriate control arm is difficult. It is also important to note that the current study involved predominantly (81\%) Asian patients with Caucasian patients comprising the remainder (19\%); further clinical studies are needed to assess whether the observed response rates are generalizable to non-Asian patient populations.

In addition to the current study, preliminary phase II results of the GEOMETRY mono-1 trial were presented at the European Society for Medical Oncology 2018 Congress (17). This multicenter, open-label, phase II study evaluated the efficacy and safety of single-agent capmatinib $400 \mathrm{mg}$ by mouth twice a day orally in adult patients with $E G F R$ and $A L K$ negative advanced NSCLC harboring $M E T$ amplification and/or MET mutations, in particular MET exon-14 skipping. ORR in treatment-naive patients $(\mathrm{n}=25)$ was a quite impressive $72 \%$ and ORR in previously treated patients $(\mathrm{n}=69)$ was $39 \%$. Interestingly, the duration of response was not evaluable yet by the time of analysis, suggesting that many responses were durable, however; specific PFS data are still pending. There is also preliminary phase Ib/II data with another selective MET inhibitor, tepotinib, in combination with gefitinib compared to a control arm of pemetrexed plus cisplatin or carboplatin in patients with EGFR-mutated NSCLC with acquired resistance to EGFR TKI therapy that was recently reported (18). Unfortunately, enrollment was terminated early due to difficulty accruing patients with MET-positive disease, defined as the presence of $M E T$ amplification by FISH or a MET IHC score of either $2+$ or $3+$. Median PFS was similar for the patients $(n=31)$ assigned to oral tepotinib $500 \mathrm{mg}$ daily plus gefitinib $250 \mathrm{mg}$ per day (dose defined during phase I portion of study) compared to those $(n=24)$ assigned to pemetrexed plus cisplatin or carboplatin with a nonsignificant hazard ratio (HR) of 0.71 . Subgroup analysis of patients with a MET IHC score of $3+(n=34)$ demonstrated that the combination of tepotinib and gefitinib was associated with a median PFS of 8.3 versus 4.4 months for chemotherapy and an HR for progression or death of 0.35 , as well as an ORR of $68 \%$ versus $33 \%$ for chemotherapy; for patients in the MET-amplified subgroup $(n=19)$, even more promisingly the combination of tepotinib and gefitinib had a median PFS of 21.2 months compared to 4.2 months with chemotherapy with a HR of 0.17 , and an ORR of $67 \%$ versus $43 \%$ for chemotherapy; however very small numbers in these groups make it impossible to reach definitive conclusions. Results of several other studies ongoing in this area are awaited (Table 1).

Combinatorial approaches with molecularly targeted therapies will continue to refine the pursuit of personalized medicine in appropriate subsets of NSCLC patients. 
While the current study by itself might not yield a practical route to an FDA indication for capmatinib, the promising data presented in the recent GEOMETRY mono-1 study suggest that capmatinib merits further clinical investigation and the current study certainly provides hope for such a combination strategy for well-selected patients, particularly with high MET GCN. Recent clinical data with tepotinib may spur further clinical development as well. More questions remain regarding the clinical efficacy of various selective MET inhibitors and the potential impact of including osimertinib as the EGFR TKI of choice in clinical studies, but more fundamental insights into the complex mechanisms governing acquired resistance involving the MET/HGF axis will likely be needed to dramatically improve clinical impact.

\section{Acknowledgements}

None.

\section{Footnote}

Conflicts of Interest: The authors have no conflicts of interest to declare.

\section{References}

1. Maroun CR, Rowlands T. The Met receptor tyrosine kinase: a key player in oncogenesis and drug resistance. Pharmacol Ther 2014;142:316-38.

2. Engelman JA, Zejnullahu K, Mitsudomi T, et al. MET amplification leads to gefitinib resistance in lung cancer by activating ERBB3 signaling. Science 2007;316:1039-43.

3. Gusenbauer S, Vlaicu P, Ullrich A. HGF induces novel EGFR functions involved in resistance formation to tyrosine kinase inhibitors. Oncogene 2013;32:3846-56.

4. Gelsomino F, Facchinetti F, Haspinger ER, et al. Targeting the MET gene for the treatment of non-small-cell lung cancer. Crit Rev Oncol Hematol 2014;89:284-99.

5. Sequist LV, Waltman BA, Dias-Santagata D, et al. Genotypic and histological evolution of lung cancers acquiring resistance to EGFR inhibitors. Sci Transl Med 2011;3:75ra26.

6. Minari R, Bordi P, Tiseo M. Third-generation epidermal growth factor receptor-tyrosine kinase inhibitors in T790M-positive non-small cell lung cancer: review on emerged mechanisms of resistance. Transl Lung Cancer Res 2016;5:695-708.
7. Yu HA, Arcila ME, Rekhtman N, et al. Analysis of tumor specimens at the time of acquired resistance to EGFR-TKI therapy in 155 patients with EGFR-mutant lung cancers. Clin Cancer Res 2013;19:2240-7.

8. Bean J, Brennan C, Shih JY, et al. MET amplification occurs with or without T790M mutations in EGFR mutant lung tumors with acquired resistance to gefitinib or erlotinib. Proc Natl Acad Sci U S A 2007;104:20932-7.

9. Shi P, Oh YT, Zhang G, et al. Met gene amplification and protein hyperactivation is a mechanism of resistance to both first and third generation EGFR inhibitors in lung cancer treatment. Cancer Lett 2016;380:494-504.

10. Deng L, Kiedrowski LA, Ravera E, et al. Response to Dual Crizotinib and Osimertinib Treatment in a Lung Cancer Patient with MET Amplification Detected by Liquid Biopsy Who Acquired Secondary Resistance to EGFR Tyrosine Kinase Inhibition. J Thorac Oncol 2018;13:e169-72.

11. York ER, Varella-Garcia M, Bang TJ, et al. Tolerable and Effective Combination of Full-Dose Crizotinib and Osimertinib Targeting MET Amplification Sequentially Emerging after T790M Positivity in EGFR-Mutant NonSmall Cell Lung Cancer. J Thorac Oncol 2017;12:e85-8.

12. Underiner TL, Herbertz T, Miknyoczki SJ. Discovery of small molecule c-Met inhibitors: Evolution and profiles of clinical candidates. Anticancer Agents Med Chem 2010;10:7-27.

13. Eathiraj S, Palma R, Volckova E, et al. Discovery of a novel mode of protein kinase inhibition characterized by the mechanism of inhibition of human mesenchymal-epithelial transition factor (c-Met) protein autophosphorylation by ARQ 197. J Biol Chem 2011;286:20666-76.

14. Liu X, Wang Q, Yang G, et al. A novel kinase inhibitor, INCB28060, blocks c-MET-dependent signaling, neoplastic activities, and cross-talk with EGFR and HER3. Clin Cancer Res 2011;17:7127-38.

15. Wu YL, Yang JC, Kim DW, et al. Safety and efficacy of INC280 in combination with gefitinib (gef) in patients with EGFR-mutated (mut), MET-positive NSCLC: a single-arm phase Ib/II study. J Clin Oncol 2014;32:abstr 8017.

16. Wu YL, Zhang L, Kim DW, et al. Phase Ib/II Study of Capmatinib (INC280) Plus Gefitinib After Failure of Epidermal Growth Factor Receptor (EGFR) Inhibitor Therapy in Patients With EGFR-Mutated, MET FactorDysregulated Non-Small-Cell Lung Cancer. J Clin Oncol 2018. [Epub ahead of print].

17. Wolf J, Seto T, Han JY, et al. Results of the GEOMETRY mono-1 phase II study for evaluation of the MET inhibitor capmatinib (INC280) in patients (pts) with MET $\mathrm{ex} 14$ 
mutated advanced non-small cell lung cancer (NSCLC). ESMO 2018 meeting, 2018:abstract LBA52.

18. Cheng Y, Zhou, Lu S, et al. Phase 2 study of tepotinib

Cite this article as: Ko B, Halmos B. Capmatinib and gefitinib combination therapy: will EGFR-mutated MET-dysregulated NSCLC “capitulate”? Transl Lung Cancer Res 2018;7(Suppl 4):S321-S325. doi: 10.21037/tlcr.2018.12.05 plus gefitinib in MET-positive epidermal growth factor receptor-mutant NSCLC. ESMO 2018 meeting, 2018:abstract 1337O. 